\title{
Laparoscopy-Assisted PEC Tubes: A Novel Treatment for Sigmoid Volvulus
}

\author{
Erika Simmerman, DO, Andrew Simmerman, BS, Randi Lassiter, MD, Asif Talukder, MD, \\ Brian Lane, MD \\ Department of Surgery, Augusta University, Augusta, Georgia, USA (all authors).
}

\begin{abstract}
Introduction: In the United States, sigmoid volvulus is frequently seen in the elderly and neurologically impaired, driving a search for less invasive but effective treatments that may obviate the need for resection. This case study describes a novel technique for sigmoidopexy: a modified endoscopic approach for percutaneous endoscopic colostomy (PEC) tube placement in combination with laparoscopy for safety.

Case Description: This is a case study reporting a novel technique. The subject was a 55-year-old man with a history of longstanding constipation and recurrent sigmoid volvulus. Because of religious beliefs, the family refused any surgical procedures requiring bowel resection. Thus, alternative methods for definitive treatment were sought. The patient underwent laparoscopic assisted PEC tube placement with placement of three colostomy tubes for sigmoidopexy. The procedure was tolerated well, and the patient had an uneventful postoperative course. With persistent symptoms of constipation and dysmotility, the colon could be vented and irrigated via the colostomy tubes. Two years after surgery the patient has all 3 PEC tubes in place without complication or recurrence of sigmoid volvulus.
\end{abstract}

Conclusion: PEC tubes have been used for sigmoidopexy with various levels of success in the treatment of recurrent volvulus, but laparoscopic assistance in placement of 3 PEC tubes has not been documented. Laparoscopic assistance allows for reduction of volvulus and decreases complications associated with PEC tube placement. This case demonstrates a novel, safe technique for the treatment of sigmoid volvulus.

Key Words: Colopexy, Percutaneous endoscopic colostomy tube, Sigmoid volvulus, Sigmoidopexy.

Citation Simmerman E, Simmerman A, Lassiter R, Talukder A, Lane B. Laparoscopy-assisted PEC tubes: a novel treatment for sigmoid volvulus. CRSLS e2016.00087. DOI: $10.4293 /$ CRSLS.2016.00087.

Copyright (C) 2017 by SLS, Society of Laparoendoscopic Surgeons. This is an open-access article distributed under the terms of the Creative Commons Attribution-Noncommercial-ShareAlike 3.0 Unported license, which permits unrestricted noncommercial use, distribution, and reproduction in any medium, provided the original author and source are credited.

Disclosures: none reported.

Address correspondence to: Erika Simmerman, DO, Department of Surgery, Augusta University, 1120 15th Street, Augusta, GA 30912, USA. Telephone: 919-601-0379, Fax: 706-721-1047, E-mail: esimmerman@augusta.edu

\section{INTRODUCTION}

Colonic volvulus is a type of malrotation in which a section of the colon twists around its mesenteric attachment site, resulting in bowel obstruction, ischemia, or both. The most common site of colonic volvulus is the sigmoid colon, accounting for $40-60 \%$ of total volvulus occurrences in most series. ${ }^{1,2}$ Patients typically present with abdominal pain, vomiting, and a distended and tympanic abdomen. ${ }^{3,4}$

In the United States, volvulus is relatively rare, accounting for only 1.9\% of bowel obstructions nationwide from 2002 through 2010.5 However, several factors have been linked to higher rates of sigmoid volvulus, including redundant sigmoid, narrow mesenteric attachments, old age, and debilitating neurological disorders (such as Parkinson's disease), although the precise etiology for the latter 2 is unknown. ${ }^{6-10}$ Because many patients exhibit volvulus in conjunction with a history of constipation or intestinal pseudo-obstruction, colonic dysmotility has been suggested as an underlying cause of severe volvulus. $3,6,11$

With sigmoid volvulus frequently seen in the elderly and neurologically impaired, it is not uncommon to pursue less invasive but effective techniques that may obviate the 
need for resection. The subject of this case study matches many of the above descriptors in that the patient was a bedridden and institutionalized 55-year-old man with a history of recurrent sigmoid volvulus. We describe a novel technique for sigmoidopexy: a modified endoscopic approach for placement of a PEC tube with a combined laparoscopic approach for safety.

\section{CASE REPORT}

A 55-year-old African-American man with a history of chronic constipation and recurrent sigmoid volvulus presented to the emergency department with increased abdominal distention, nausea, and vomiting. He had been admitted 2 months earlier for a bleeding duodenal ulcer and recurrent sigmoid volvulus that was decompressed with rigid proctoscopy and rectal tube placement by the Department of Gastrointestinal Medicine. His medical history was significant for a cerebrovascular accident, leaving the patient neurologically devastated, requiring institutionalization, as the patient was nonverbal and confined to bed. His surgical history was significant for tracheostomy, gastrostomy tube placement, and multiple episodes of endoscopic decompression of sigmoid volvulus.

On presentation, the patient's vital signs were stable. Significant physical examination findings were severe ab- dominal distention with tympany, tenderness to palpation without peritoneal signs, and a gastrostomy tube in place, draining bilious fluid. An abdominal radiograph demonstrated findings consistent with sigmoid volvulus with obstruction (Figure 1A). CT of the abdomen and pelvis demonstrated redundant and dilated sigmoid colon to the level of the descending colon, causing compression of the liver, gallbladder, stomach, and duodenum, but no free air or fluid to suggest perforation (Figure 1B, 1C).

The patient was admitted to the Department of Surgery and underwent emergent decompressive colonoscopy followed by placement of a rectal tube with successful reduction of the sigmoid volvulus. At this time extensive discussions took place with the family (as the patient was nonverbal) regarding more definitive treatment for recurrent sigmoid volvulus. The family adamantly refused any sort of bowel resection, and alternative treatments were therefore discussed.

On hospital day 2, the patient underwent laparoscopyassisted PEC tube placement for sigmoidopexy. Both the laparoscopic and endoscopic parts of the procedure were performed by a surgeon experienced in minimally invasive procedures with additional training in endoscopy. The use of laparoscopy enabled complete detorsion of the sigmoid colon at the initiation of the procedure and place-

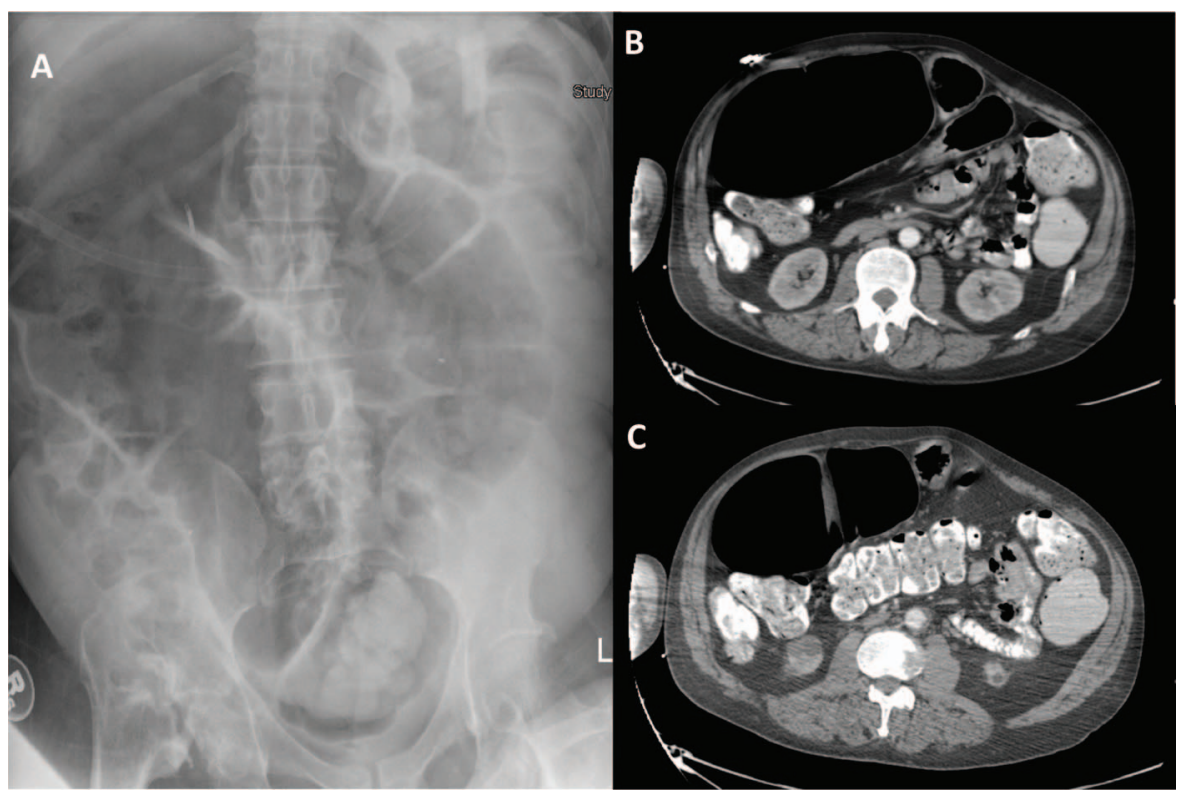

Figure 1. A, Abdominal X-ray demonstrating a distal colon grossly distended with an appearance highly suggestive of a sigmoid volvulus with obstruction. B, C, CT abdomen/pelvis with findings of a redundant dilated sigmoid colon to the level of the descending colon, compressing adjacent structures including the liver, gallbladder, stomach, and duodenum. No free air or fluid to suggest perforation. 
ment in correct anatomic position. Three PEC tubes were then placed for sigmoidopexy under direct laparoscopic visualization in addition to endoscopic visualization (Figures 2-5). The tubes were placed by introduction of the endoscope with advancement to the level of the sigmoid colon where the first tube was to be placed. A point on the abdominal wall was selected, and a needle was introduced through the abdominal wall into the colon and the catheter over needle was passed into the colon. The endoscopist placed the snare through the channel of the endoscope into the colon, the looped guidewire was passed through the catheter into the colon and caught by

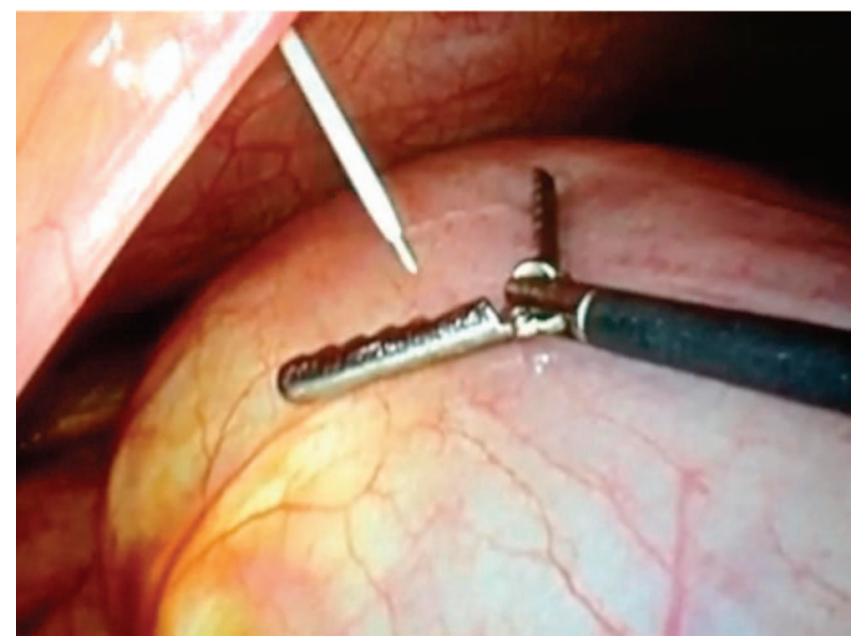

Figure 2. Laparoscopic view of PEC tube placement. In this step the access needle has been introduced percutaneously through the abdomen and is observed to enter the sigmoid colon.

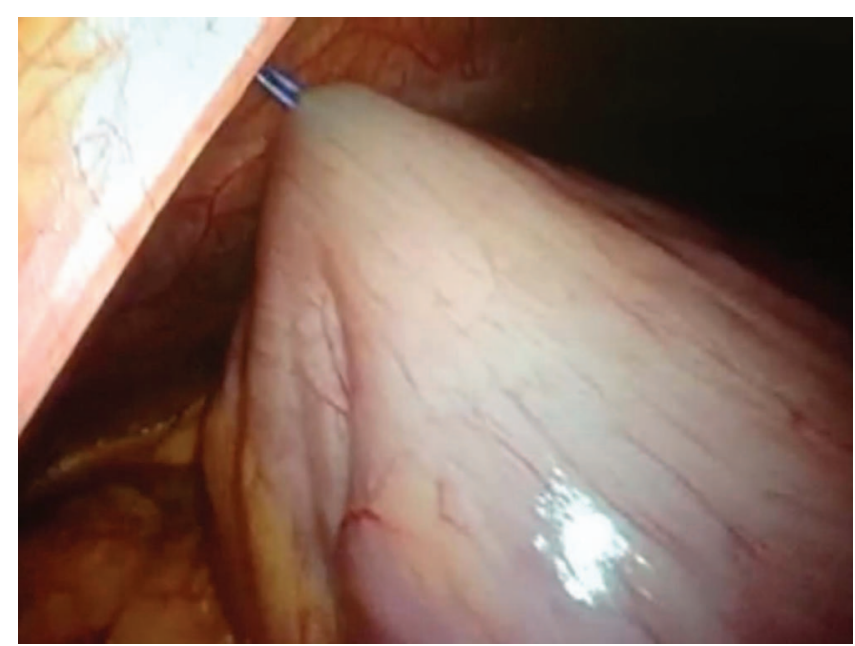

Figure 3. Laparoscopic view of PEC tube placement. In this step the percutaneous gastrostomy tube device is being pulled through the colon. the snare, which was then pulled out along with the endoscope and released by the snare. The PEC tube was then secured to the looped end of the guidewire, and the endoscope and tube were brought back through the abdominal wall by pulling on the guidewire, visualizing the internal bumper sitting against the colonic mucosa. The procedure was repeated 3 times for each tube placement in the sigmoid colon with direct extraluminal visualization to avoid undue tension or torsion of the colon or place-

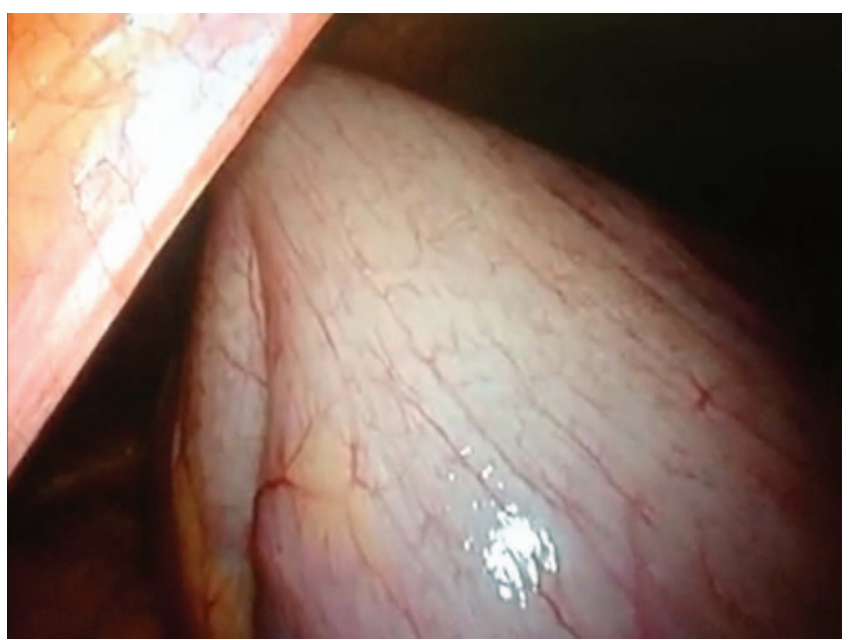

Figure 4. Laparoscopic view of PEC tube placement. In this step the PEC tube device has been pulled through the colon and out of the abdominal wall, and the sigmoid colon is being secured to the abdominal wall.

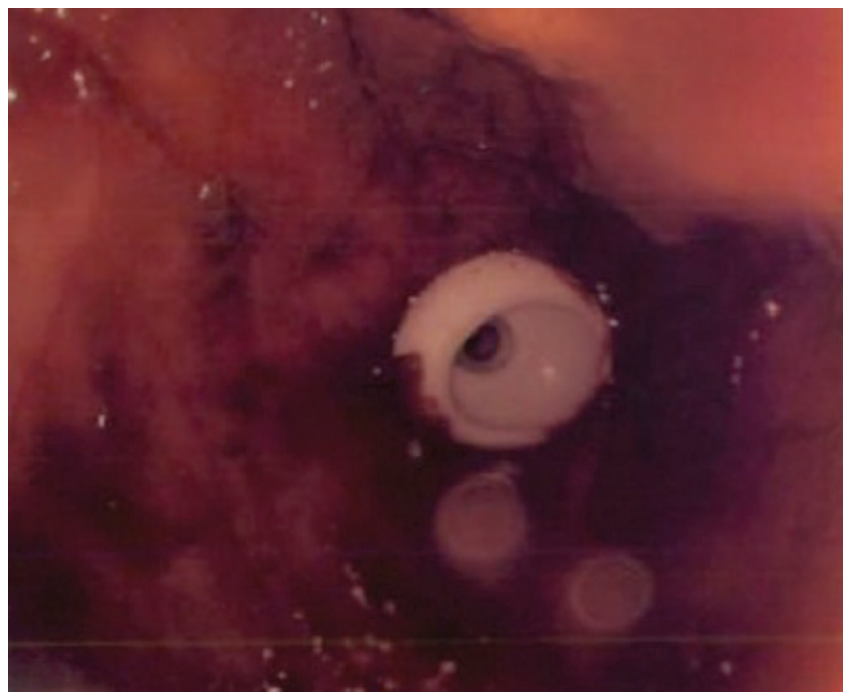

Figure 5. Endoscopic view of PEC tube placement. In this final step the PEC tube device has been pulled through the colon and out the abdominal wall and adequate fixation is visualized with appropriate pressure within the colon wall. 
ment of the tubes through other vital structures. The procedure was tolerated well and the patient recovered on the surgical floor with an uneventful postoperative course.

The patient had persistent symptoms of colonic dysmotility. However, as an added benefit of the procedure, the colon was able to be vented and irrigated via the 3 PEC tubes. Two years after surgery, the patient still had all PEC tubes in place without complication or recurrent volvulus.

\section{DISCUSSION}

Treatment for individuals with acute sigmoid volvulus but no evidence of ischemia or peritonitis starts with endoscopic detorsion. However, because of the relatively high rate of recurrence (with estimates as high as 60-70\%), the definitive treatment is bowel resection after colonic decompression. ${ }^{12-14}$ Because of the invasive nature of the bowel resection procedure, other long-term treatments have been explored. In the case of our patient, the family refused any major operation that would require removal of internal organs for religious reasons. However, because of the history of recurrence, a long-term treatment was necessary. Therefore sigmoidopexy was performed, using 3 PEC tubes. PEC tubes were used for fixation instead of sutures, which have previously been shown to be ineffective for sigmoidopexy. ${ }^{15}$

This case demonstrates the ability to successfully augment the purely endoscopic approach by using a laparoscope. This technique facilitated the correction of the volvulus and return of the redundant sigmoid colon back to its anatomic position and aided in protecting the percutaneous colostomy tube placement versus placement by use of endoscopy alone, which may cause other drastic complications associated with tension, torsion, or inadvertent placement through unintended structures. This procedure used several techniques that have not yet gained popularity. Use of the laparoscope was unusual, despite observations that instrumental perforation is a complication in more than $1 \%$ of endoscopic decompression cases. ${ }^{15-19}$ Not only could the laparoscope reduce risk of complication during the decompression phase, its presence during PEC placement (combined with the endoscope) allowed for more informed movements both inside and outside of the bowel. The primary question is therefore whether the increased frequency of laparoscopy-related complications and complications associated with general anesthesia will outweigh the reduction in complications by affording better visualization and instrument control, a question that can only be answered in a much broader study. This procedure could also have a potential application in the setting of an intensive care unit with moderate sedation, given minimal the manipulation required with the laparoscope; however, from our limited experience we would still recommend performing the procedure in a controlled setting such as the operating room. The patient in this case demonstrated no adverse effects from the laparoscopy.

The placement of extra PEC tubes, in our case placing 3 tubes instead of 1 or 2 creates additional opportunities for complications, but may help to lower the rate of recurrence, which can be quite high when only 1 tube is used. ${ }^{20-23}$ In a study of an individual initially fitted with 2 tubes, recurrence was observed only after the removal of 1 tube and was not observed again after the tube was replaced. ${ }^{24}$ Placement of 2 colostomy tubes creates an effective 3-point fixation, although successful sigmoidopexies have been performed in the past with 3 and even 4 tubes. ${ }^{20,25,26}$ Because volvulus is often seen in conjunction with a damaged or stretched mesentery, reliance on the mesentery as an adequate point of fixation may be misguided. The use of 3 intraluminal tubes could therefore be most effective for long-term therapy. ${ }^{15,27}$

More longitudinal data are necessary to provide an accurate recurrence rate, and a much broader study is necessary to evaluate the incidence of complications. However, this case demonstrated the safety and feasibility of performing a sigmoidopexy, with a novel technique: laparoscopy-assisted PEC tube placement with 3-tube fixation. (Figure 6).

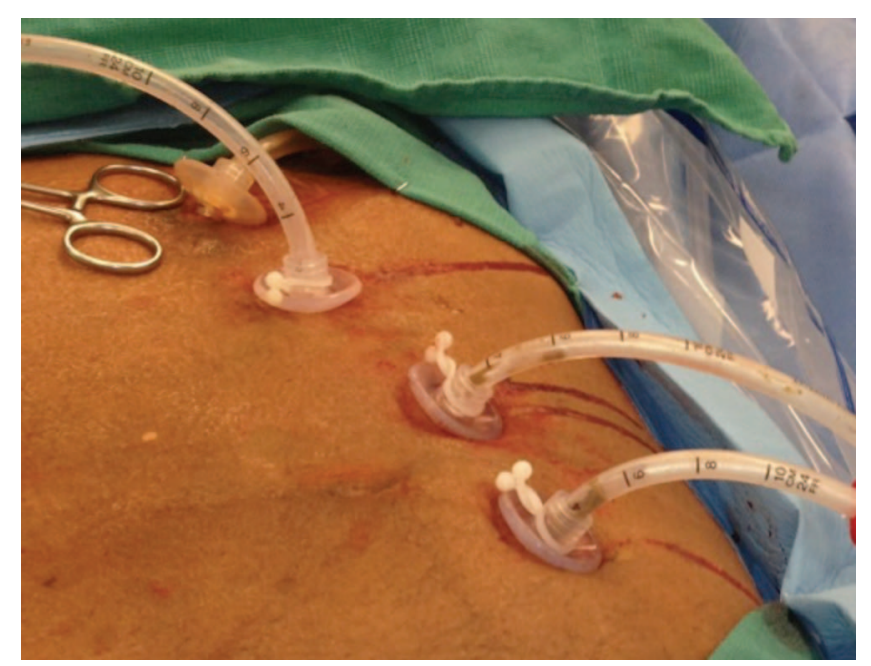

Figure 6. External view of the 3 PEC tubes at the completion of the procedure. 


\section{References:}

1. Ballantyne GH. Review of sigmoid volvulus: history and results of treatment. Dis Colon Rectum 1982;25:494-501.

2. Påhlman L, Enblad $\mathrm{P}$, Rudberg $\mathrm{C}, \mathrm{Krog} \mathrm{M}$. Volvulus of the colon. a review of 93 cases and current aspects of treatment. Acta Chirurg Scand. 1989;155:53-56.

3. Atamanalp SS, Yildirgan MI, Basoglu M, et al. Clinical presentation and diagnosis of sigmoid volvulus: outcomes of 40-year and 859-patient experience. J Gastroenterol Hepatol. 2009;24:1154.

4. Atamanalp SS, Yildirgan MI, Başoğlu M, Kantarci M, Yilmaz I. Sigmoid colon volvulus in children: review of 19 cases. Pediatr Surg Int. 2004;20:492-495.

5. Halabi WJ, Jafari MD, Kang CY, et al. Colonic volvulus in the United States: trends, outcomes, and predictors of mortality. Ann Surg 2014;259:293-301.

6. Toebosch S, Tudyka V, Masclee A, Koek G. Treatment of recurrent sigmoid volvulus in Parkinson's disease by percutaneous endoscopic colostomy. World J Gastroenterol. 2012;18: $5812-5815$.

7. Rosenthal MJ, Marshall CE. Sigmoid volvulus in association with Parkinsonism: report of four cases. J Am Geriatr Soc. 1987; 35:683-684.

8. Mangiante EC, Croce MA, Fabian TC, Moore OF 3rd, Britt LG. Sigmoid volvulus: a four-decade experience. Am J Surg. 1989; $55: 41-44$.

9. Oren D, Atamanalp SS, Aydinli B, et al. An algorithm for the management of sigmoid colon volvulus and the safety of primary resection: experience with 827 cases. Dis the Colon Rectum. 2007;50:489-497.

10. Baker DM, Wardrop PJ, Burrell H, Hardcastle JD. The management of acute sigmoid volvulus in Nottingham. $J$ R Coll Surg Edinb. 1994;39:304-306.

11. de Betue, CT. Volvulus as a complication of chronic intestinal pseudo-obstruction syndrome. Eur J Pediatr. 2011;170: 1591-1595.

12. Larkin JO, Thekiso TB, Waldron R, Barry K, Eustace PW. Recurrent sigmoid volvulus: early resection may obviate later emergency surgery and reduce morbidity and mortality. Ann $R$ Coll Surg Engl. 2009;91:205-209.

13. Tan KK, Chong CS, Sim R. Management of acute sigmoid volvulus: an institution's experience over 9 years. World J Surg. 2010;34:1943-1948.
14. Bruusgaard C. Volvulus of the sigmoid colon and its treatment. Surgery. 1947;22:466-478.

15. Armstrong DN, Ballantyne GH. Laparoscopic treatment of volvulus of the colon. In: Laparoscopic Surgery. Philadelphia: W. B. Saunders Co., 1994.

16. Arnold GJ, Nance FC. Volvulus of the sigmoid colon. Ann Surg. 1973;177:527-537.

17. Friedman JD, Odland MD, Bubrick MP. Experience with colonic volvulus. Dis Colon Rectum. 1989;32:409-16.

18. Siroospour D, Bernardi RS. Volvulus of the sigmoid colon. Dis Colon Rectum. 1976;19:535-541.

19. Påhlman L, Enblad P, Rudberg C, Krog M. Volvulus of the colon: a review of 93 cases and current aspects of treatment. Acta Chir Scand. 155:53-56, 1989.

20. Choi D, Carter R. Endoscopic sigmoidopexy: a safer way to treat sigmoid volvulus? J R Coll Surg Edinb 1968;19:64.

21. Gordon-Weeks AN, Lorenzi B, Lim J, Cristaldi M. Laparoscopic-assisted endoscopic sigmoidopexy: a new surgical option for sigmoid volvulus. Dis Colon Rectum. 2011;54:645647.

22. Ennis OW, Feroz AS, Stephenson BM. Percutaneous endoscopic colostomy: an alternative treatment of acute colonic pseudo-obstruction. Colorectal Dis. 2001;3:205.

23. Daniels IR, Lamparelli MJ, Chave H, Simson JN. Recurrent sigmoid volvulus treated by percutaneous endoscopic colostomy. Br J Surg. 2000;87:1419.

24. Al-Alawi, Ibrahim K. percutaneous endoscopic colostomy: a new technique for the treatment of recurrent sigmoid volvulus. Saudi J Gastroenterol. 2010;16:120-121.

25. Pinedo G, Kirberg A. Percutaneous endoscopic sigmoidopexy in sigmoid volvulus with T-fasteners: report of two cases. Dis Colon Rectum. 2001;44:1867-1869.

26. Gallagher HJ, Aitken D, Chapman AH, Ambrose NS. Additional experience of endoscopic T-bar sigmoidopexy. Dis Colon Rectum. 2002;45:1565-1566.

27. Akinkuotu A, Samuel JC, Msiska N, Myula C, Charles AG. The role of the anatomy of the sigmoid colon in developing sigmoid volvulus: a case-control study. Clin Anat. 2011;24:634637. 